\title{
A NOTE ON EMBEDDING A PARTIALLY ORDERED RING IN A DIVISION ALGEBRA
}

\author{
WILLIAM H. REYNOLDS
}

\begin{abstract}
If $H$ is a maximal cone of a ring $A$ such that the subring generated by $H$ is a commutative integral domain that satisfies a certain centrality condition in $A$, then there exist a maximal cone $H^{\prime}$ in a division ring $A^{\prime}$ and an order preserving monomorphism of $A$ into $A^{\prime}$, where the subring of $A^{\prime}$ generated by $H^{\prime}$ is a subfield over which $A^{\prime}$ is algebraic. Hypotheses are strengthened so that the main theorems of the author's earlier paper hold for maximal cones.
\end{abstract}

The terminology of the author's earlier paper [3] will be used. For a subsemiring $H$ of a ring $A$, we write $H-H$ for $\{x-y: x, y \in H\}$; this is the subring of $A$ generated by $H$. We say that $H$ is a u-hemiring of $A$ if $H$ is maximal in the class of all subsemirings of $A$ that do not contain a given element $u$ in the center of $A$. We call $H$ left central if for every $a \in A$ and $h \in H$ there exists $h^{\prime} \in H$ with $a h=h^{\prime} a$. Recall that $H$ is a cone if $H \cap(-H)=\{0\}$, and a maximal cone if $H$ is not properly contained in another cone.

First note that in [3, Theorem 1] the commutativity of the hemiring, established at the beginning of the proof, was only exploited near the end of the proof and was not used to simplify the earlier details. In the present paper, this fact is put to good use, for commutativity will not be at our immediate disposal. We will show that with stronger hypotheses, the conic hemiring of Theorems 1, 2, and 3 in [3] can be replaced by a maximal cone to obtain Theorems 1, 2, and 3 listed below. This is of interest since, as observed in [2], maximal cones are somewhat more plentiful than conic hemirings. Our first theorem is of a more general nature.

THEOREM A. Let $H$ be a nontrivial maximal cone of a ring $A$ such that for every nonzero $a, b \in H-H, a b$ is also nonzero. Then the following hold:

(1) If $H$ is left central in $A$, then there exist a conic 1-hemiring $H^{\prime}$ in a ring $A^{\prime}$ with 1 and an order preserving monomorphism $A \rightarrow A^{\prime}$, where every nonzero element of $H^{\prime}$ has an inverse in $H^{\prime}$ and every nonzero element of $A$ has a left inverse.

Received by the editors November 18, 1971.

AMS (MOS) subject classifications (1970). Primary 06A10, 06A70; Secondary 16A40.

Key words and phrases. Partially ordered ring, maximal cone, order preserving monomorphism, algebraic division algebra. 
(2) If in addition to the hypothesis of (1), $H$ is commutative, then $H^{\prime}$ is left central in $A^{\prime}$ and $A^{\prime}$ is a division ring.

(3) If in addition to the hypotheses of (1) and (2), $H-H=H \cup(-H)$, then $F=H^{\prime} \cup\left(-H^{\prime}\right)$ is a subfield of $A^{\prime}$ over which $A^{\prime}$ is an algebraic $F$ algebra.

(4) If $A$ is commutative and $H-H=H \cup(-H)$, then there exist a conic 1-hemiring $H^{\prime}$ in a field $A^{\prime}$ and an order preserving monomorphism $A \rightarrow A^{\prime}$, where $F=H^{\prime} \cup\left(-H^{\prime}\right)$ is a subfield of $A^{\prime}$ over which $A^{\prime}$ is algebraic.

Recall that a positive cone $H$ of a ring $A$ is archimedean if for every $a, b \in H$ with $a \neq 0$ there exists $n \in N$ such that $n a-b \in H$.

THEOREM 1. Let $H$ be a nontrivial archimedean left central maximal cone of a ring $A$ and assume that the ring $H-H$ has no proper divisors of zero. Then there exist a left central conic 1-hemiring $H^{\prime}$ in a division ring $A^{\prime}$ and order preserving monomorphisms of $A$ into $A^{\prime}$ and $H^{\prime}-H^{\prime}$ into the ring $R$ of real numbers, where $F=H^{\prime}-H^{\prime}$ is a subfield of $A^{\prime}$ over which $A^{\prime}$ is algebraic. Moreover, if $A$ is commutative, so is $A^{\prime}$.

THEOREM 2. Let $H$ be a nontrivial archimedean maximal cone of a commutative ring $A$ and assume that the ring $H-H$ has no proper divisors of zero. Then there exists an order preserving monomorphism from $A$ into the ring $C$ of complex numbers (with $R^{+}$as positive cone) which is unique on $H-H$.

THEOREM 3. Let $A$ be a ring containing $R^{+}$as a subsemiring. Then $R^{+}$is a left central maximal cone of $A$ iff $A$ is isomorphic to $R, C$, or the division ring $Q$ of real quaternions.

We now proceed to the proofs of these theorems.

Lemma. Let $H$ be a nontrivial left central maximal cone of a ring $A$. (a) If $a \notin H$, then $\sum_{i=0}^{m} h_{i} a^{i}+\sum_{i=1}^{m} n_{i} a^{i}=0$ for some $h_{0}, \cdots, h_{m} \in H$ and $n_{1}, \cdots, n_{m} \in N \cup\{0\}$.

(b) If for every nonzero $a, b \in H-H$, ab is also nonzero, then $A$ has no proper divisors of zero and $M=H \backslash\{0\}$ is a multiplicatively closed subset that is left and right cancellable.

(c) Assume that for every nonzero $a, b \in H-H$, ab is also nonzero. If for some nonzero $a \in A, \sum_{i=0}^{m} h_{i} a^{i}+\sum_{i=1}^{m} n_{i} a^{i}=0$, where $h_{0}, \cdots, h_{m} \in H$ and $n_{1}, \cdots, n_{m} \in N \cup\{0\}$, and if $m$ is minimal, then $h_{0} \neq 0$.

Proof. (a) Since $H$ is left central, $H(a)=\left\{\sum_{i=0}^{k} h_{i} a^{i}+\sum_{i=1}^{k} n_{i} a^{i}\right.$ : $h_{0}, \cdots, h_{k} \in H$ and $\left.n_{1}, \cdots, n_{k} \in N \cup\{0\}\right\}$ is a subsemiring of $A$ that properly contains $H$. Hence $H(a) \cap-H(a) \neq \varnothing$, so for some $x, y \in H(a)$, $x=-y$. Gathering all terms on the left side of the equation and introducing zero terms as needed, we obtain the desired result. 
(b) Assume $a m=0, a \in A, m \in M$. If $a+m \in H$, then $a \in H-H$, so $a=0$ by hypothesis. If $a+m \notin H$, then from (a), $\sum_{i=0}^{k} h_{i}^{\prime}(a+m)^{i}+$ $\sum_{i=1}^{k} n_{i}(a+m)^{i}=0$ for some $h_{i}^{\prime} \in H$ and $n_{i} \in N \cup\{0\}$. Since $m \neq 0$ and $H$ is conic, this can be written as $\sum_{i=0}^{k} h_{i} a^{i}+\sum_{i=1}^{k} n_{i} a^{i}=0$ for some $h_{i} \in H$, where $h_{0} \neq 0$. Multiplying on the right by $m$, we get $h_{0} m=0$, which contradicts the hypothesis. Hence $a+m \in H$. Thus, $a=0$. The proof that $m a=0$ implies $a=0$ is similar; simply multiply on the left by $m$ and use the left central hypothesis.

Now assume $x y=0$ with $x, y \in A$. Let $m \in M$. If $x+m, y+m \in H$, then $x, y \in H-H$, so we may assume $x+m \notin H$. Then as above, $\sum_{i=0}^{k} h_{i} x^{i}+$ $\sum_{i=1}^{k} n_{i} x^{i}=0$ for some $h_{i} \in H$ and $n_{i} \in N \cup\{0\}$, where $h_{0} \neq 0$. Multiplying on the right by $y$, we get $h_{0} y=0$, whence $y=0$ from above. The proof is similar if $y+m \notin H$.

It is clear that $M$ is multiplicatively closed.

To show $M$ is right cancellable, suppose $a b \in M$ and $b \in M$, but $a \notin M$. Then $a \notin H$, so $\sum_{i=0}^{m} h_{i} a^{i}+\sum_{i=1}^{m} n_{i} a^{i}=0$ for some $h_{i} \in H$ and $n_{i} \in N \cup\{0\}$. Since $a b \in H$ and $H$ is left central, by induction one shows that for $1 \leqq i \leqq m, a^{i} b^{m}=g_{i} b^{m-i}$ for some $g_{i} \in H$. Thus

$$
\sum_{i=0}^{m} h_{i} g_{i} b^{m-i}+\sum_{i=1}^{m} n_{i} g_{i} b^{m-i}=0 b^{m}=0 .
$$

Since $A$ has no zero divisors, one shows that either $h_{i} g_{i} b^{m-i} \neq 0$ or $n_{i} g b^{m-i} \neq 0$ for some $i=1, \cdots, m$. But since terms of this sort belong to $H$, the above equation contradicts the fact that $H$ is conic. Hence, $a \in M$. Therefore, $M$ is right cancellable. The proof that $M$ is left cancellable is similar.

(c) Suppose $m$ is minimal and $h_{0}=0$. Multiplying on the left by any nonzero $h \in H$, we get

$$
\left(\sum_{i=1}^{m}\left(h h_{i}+n_{i} h\right) a^{i-1}\right) a=0
$$

Hence by (b), $\sum_{i=1}^{m}\left(h h_{i}+n_{i} h\right) a^{i-1}=0$, which contradicts the minimality of $m$. Thus, $h_{0} \neq 0$.

Proof of Theorem A. Using part (b) of the Lemma, one shows as in the proof of [3, Theorem 1] that $A^{\prime}=\{a \mid b: a \in A, b \in M\}$ is a ring with identity 1 and $a \mapsto b a / b$ is a monomorphism $\theta: A \rightarrow A^{\prime}$ such that for each $b \in M, \theta(b)$ is invertible in $A^{\prime}$. One easily checks that $H^{\prime}=\left\{a \mid b \in A^{\prime}: a \in H\right\}$ is a cone in $A^{\prime}$ containing 1 , every nonzero element of $H^{\prime}$ has an inverse in $H^{\prime}$, and $\theta: A \rightarrow A^{\prime}$ preserves order.

Now let $x / y \in A^{\prime} \backslash H^{\prime}$. Then $x \notin H$, so $\sum_{i=0}^{m} h_{i} x^{i}+\sum_{i=1}^{m} n_{i} x^{i}=0$ for some $h_{i} \in H$ and $n_{i} \in N \cup\{0\}$. Assume $m$ is minimal so that by (c) of the Lemma, 
$h_{0} \neq 0$. Now by definition of the operations in $A^{\prime}$, it follows that $(x / y)^{i}=$ $x^{i} \mid f_{i}$ for some $f_{i} \in M$, and

$$
\begin{aligned}
\frac{h_{0}^{2}}{h_{0}}+\sum_{i=1}^{m} \frac{h_{0} h_{i} f_{i}}{h_{0}}\left(\frac{x}{y}\right)^{i}+\sum_{i=1}^{m} \frac{n_{i} h_{0} f_{i}}{h_{0}}\left(\frac{x}{y}\right)^{i} & =h_{0}\left(h_{0}+\sum_{i=1}^{m} h_{i} x^{i}+\sum_{i=1}^{m} n_{i} x^{i}\right) / h_{0} \\
& =h_{0}(0) / h_{0}=0 .
\end{aligned}
$$

Hence $\sum_{i=1}^{m} h_{i}^{\prime}(x / y)^{i}=-h_{0}^{2} / h_{0}$, where $h_{i}^{\prime}=h_{0} h_{i} f_{i} / h_{0}+n_{i} h_{0} f_{i} / h_{0} \in H^{\prime}, i=1$, $\cdots, m$. Since $h_{0} \neq 0, h_{0}^{2} / h_{0}$ has an inverse $h_{0}^{-1} \in H^{\prime}$, so

$$
\sum_{i=1}^{m} h_{0}^{-1} h_{i}^{\prime}\left(\frac{x}{y}\right)^{i}=-1 .
$$

Thus $H^{\prime}$ is a 1 -hemiring of $A^{\prime}$. Also $x / y$ has $-\sum_{i=1}^{m} h_{0}^{-1} h_{i}^{\prime}(x / y)^{i-1}$ as left inverse, so every element of $A^{\prime}$ has a left inverse.

(2) As in the proof of [3, Theorem 1], the commutativity of $H$ implies that $H^{\prime}$ is left central in $A^{\prime}$, and part (b) of the Lemma implies that $A^{\prime}$ is a division ring.

(3) Since $H-H=H \cup(-H)$, one easily checks that $F=H^{\prime} \cup-H^{\prime}$ is a subfield of $A^{\prime}$. As in the proof of [3, Theorem 1], $F$ is contained in the center of $A^{\prime}$. The result follows.

(4) This is an immediate result of (3) and the definition of multiplication in $A^{\prime}$.

Definition. Let $H$ be a cone of a ring $A$ and let $u \in H$ commute with every element of $H$. We say that $u$ is a weak $H$-unit if for every $a \in H$ there exists $n \in N$ such that $n u-a \in H$.

Proof of Theorem 1. By Theorem $\mathrm{A}$, there exist a conic 1-hemiring $H^{\prime}$ in a ring $A^{\prime}$ with 1 and an order preserving monomorphism $A \rightarrow A^{\prime}$, where every nonzero element of $H^{\prime}$ has an inverse in $H^{\prime}$. Let $a / b \in A^{\prime}$. As in the proof of [3, Theorem 1], since $H$ is archimedean and $b$ is a nonzero element of $H, n b-a \in H$ for some $n \in N$, so $n 1-a / b=(n b-a) / b \in H^{\prime}$, whence 1 is a weak $H^{\prime}$-unit. Thus by [1, Proposition 7], there exists an order preserving monomorphism $H^{\prime}-H^{\prime} \rightarrow R$. In particular, this implies that $H$ is commutative and $H-H=H \cup(-H)$. The result now follows from (3) and (4) of Theorem A.

Theorem 2 follows from Theorem 1 just as in [3].

In Theorem 3, necessity is an immediate result of Theorem 1 and Frobenius' classical result on real division algebras. Since every conic hemiring is a maximal cone, sufficiency follows at once from [3, Theorem 3].

In Theorems 1 and 2 it was hoped that as in the corresponding theorems of [3], it would suffice for $H$ to be archimedean with respect to a single element, that is for $H$ to contain a weak $H$-unit $u$. But as the following 
example shows, a stronger requirement is needed in order to embed $H-H$ in $R$ as an ordered subring.

EXAMPLE. Let $A$ be the subring of $C[0,1]$ generated by the constant function 1 and the identity function $I$. Then $A=\left\{\sum_{i=0}^{m} n_{j} I^{j}: n_{j} \in Z, m\right.$ a nonnegative integer $\}$. Let $P=\{f \in A: f(x) \geqq 0$ for all $x \in[0,1]\}$. Then $P$ is a cone and for every $f \in A, n l-f \in P$ for some $n \in N$. It is easy to see that $A$ has no proper divisors of zero. Now by Zorn's lemma $P$ is contained in a maximal cone $H$. Then $H-H$ has no divisors of zero and for every nonzero $f \in H, n l-f \in P \subseteq H$ for some $n \in N$, and $H$ is left central since $C[0,1]$ is commutative. However any embedding of $H-H$ into $R$ as an ordered subring would yield a similar embedding of $P-P$ into $R$. But no such embedding can exist since $g^{2}$ is a nonzero element of $P$, but no $n \in N$ exists such that $n g^{2}-g \in P$.

\section{REFERENCES}

1. D. K. Harrison, Finite and infinite primes for rings and fields, Mem. Amer. Math Soc. No. 68 (1966). MR 34 \#7550.

2. C. W. Kohls and W. H. Reynolds, Embedding rings with a maximal cone and rings with an involution in quaternion algebras, Trans. Amer. Math. Soc. (to appear.)

3. W. H. Reynolds, Embedding a partially ordered ring in a division algebra, Trans. Amer. Math. Soc. 158 (1971), 293-300.

Department of Mathematics, State University of New York, College at Cortland, Cortland, New York 13045 